\title{
Il Libro del Deuteronomio come fonte teologica della dimensione anamnetica dell'anno liturgico
}

\section{Prefazione}

Tra gli antichi popoli, in Estremo Oriente, l'arte della scrittura non era un'abilità comunemente conosciuta e alla portata di tutti, pertanto la memoria umana aveva molta importanza e significato. In pratica, questo significava che essa era responsabile della trasmissione fedele degli eventi storici alle generazioni successive. A causa del singolare significato e del modo dell'uso della memoria nell'esperienza umana, le nozioni di "ricordare", "memoria" e "memoriale" hanno assunto nella Sacra Scrittura un significato teologico.

Nella Sacra Scrittura "ricrodarsi di qualcuno", "ricordare qualcuno", non significava un'attività puramente intellettuale, attraverso la quale veniva richiamata alla memoria una persona. Il ricordarsi di qualcuno implicava anche delle azioni concrete a favore di quella persona ${ }^{2}$. La Bibbia parla della memoria di Dio che si ricorda dell'uomo e della memoria dell'uomo che si ricorda di Dio. Tutti questi ricordi bilaterali sono legati a degli eventi concreti del passato, quando l'uomo entrava in relazione con Dio.

${ }^{1}$ Don Dariusz Kwiatkowski prof. UAM dr hab. - sacerdore della diocesi di Kalisz; professore presso la Facoltà di Teologia dell'Università di Adam Mickiewicz a Poznań. Direttore della Sezione di Teologia pratica. Il principale lavoro di ricerca riguarda la liturgia dei sacramenti rinnovata al Concilio Vaticano II e all'anno liturgico; indirizzo: e-mail: darkwt63@amu.edu.pl. ORCID: 0000-0002-8014-0016.

${ }^{2}$ In un certo senso, il significato del termine "ricordarsi di qualcuno" è presente nel nostro pensiero. In alcuni casi, chiedendo a qualcuno di ricordarsi di una persona, s'intende di intraprendere alcune azioni per favorire la persona che vuole ricordare. 
Lo scopo dell'articolo è quello di mostrare la dimensione anamnetica delle celebrazioni dell'anno liturgico. Per raggiungere l'obiettivo prefissato, prima verrà mostrata in modo sintetico la dimensione anamnetica del celebrare, presentata nel Libro del Deuteronomio, chiamato "libro della memoria". Nella seconda parte dell'articolo, verrà spiegata la celebrazione dell'anno liturgico come l'anamnesi degli eventi salvifici nella vita di Cristo.

Il tema della dimensione anamnetica dell'anno liturgico è stato discusso molte volte nella letteratura liturgica polacca. Questa problematica è stata affrontata in un contesto più ampio tra gli altri nella ricerca scientifica di: don Waldemar Pałęcki e don Daniel Brzeziński. È anche uscita un'opera collettiva, curata da don Jacek Nowak dal titolo Il mistero di Cristo nell'anno liturgico, pubblicata dall'Hlondianum di Poznań nel 2012. Nel mio articolo voglio presentare la fonte teologica veterotestamentaria della dimensione anamnetica dell'anno liturgico. Senza dubbio la fonte primaria per il presente studio è il Libro del Deuteronomio. Per raggiungere l'obiettivo della ricerca, l'articolo utilizzerà il metodo storico-critico e il metodo dell'ermeneutica liturgica.

\section{Dimensione anamnetica delle celebrazioni nella Sacra Scrittura}

La Costituzione sulla liturgia, dando una definizione descrittiva della liturgia, la colloca in una fase specifica della storia della salvezza. Ricorda anche che l'Antico Testamento prefigurava e preparava la "pienezza del tempo" che si adempì in Gesù Cristo ${ }^{3}$. Pertanto, le radici della liturgia cristiana vanno ricercate nella liturgia ebraica. Non può essere diversamente nel caso delle festività cristiane che molto spesso sono direttamente collegate alle festività del popolo eletto. Il tema delle celebrazioni nell'Antico Testamento è molto ampio e sfaccettato. Per raggiungere l'obiettivo di ricerca prefissato, occorre concentrarsi sulla dimensione anamnetica delle festività ebraiche. Questa dimensione delle celebrazioni è dimostrata in particolare nel Libro del Deuteronomio, il quale parla del passato del popolo eletto come di una realtà presente e attuale nella contemporaneità. Il verbo "ricordare" esprime qui la salvezza che si rende presente nel culto. Si può dire giustamente che il passato genera il presente dell'uomo e lo orienta verso il futuro: l'uomo è soprattutto la sua storia. La rivelazione biblica presenta questa storia, come una storia d'amore, una storia dell'alleanza tra Dio e l'uomo. Il Dio dell'alleanza dona sempre all'uomo il suo infinito amore, e per questo l'uomo dovrebbe ricordarsi del suo Dio. Il ricordo di Dio riguardo al suo popolo dovrebbe naturalmente includere la memoria

3 Cfr. Concilio Vaticano II, La Costituzione sulla Sacra Liturgia Sacrosanctum Concilium, 1-7, Città del Vaticano 2002 (citata come: CL). 
delle grandi opere di Dio realizzate per il popolo eletto. Tuttavia, nonostante le esortazioni di Dio stesso, il popolo si dimentica molto spesso del suo Dio (cfr. Dt 4,$9 ; 8,11 ; 9,7)^{4}$.

Nella Sacra Scrittura troviamo molto spesso un richiamo a ricordarsi. Questo appello è bidirezionale, perché da un lato le persone fanno richiamo alla memoria di Dio e Dio si appella alla memoria del popolo. Indubbiamente, esistono più richiami da parte di Dio alla memoria di Israele, perché tenendo presente la sua elezione, torni al "proprio cuore" e si converta. L'uomo dovrebbe "esercitare" la sua memoria costantemente e, prestando attenzione al numero delle testimonianze, si può affermare che questo era il tratto più caratteristico della pietà veterotestamentaria. In modo particolare lo sottolinea il Libro del Deuteronomio, il quale contiene "la teologia della memoria"s.

Analogamente, come l'ebreo $z k r$ e i derivati zākar e zikkārōn, così pure la traduzione greca dei termini anāmnesis e mimnēskomai vanno intesi in senso attivo e dinamico ${ }^{6}$. Significano un'azione con la quale viene richiamata alla memoria una cosa o una persona. L'anāmnesis liturgica dell'Antico Testamento quindi non è una memoria per ricordare oggetti, puramente intellettuale, ma è un fatto soggettivo, un'azione che si esprime in gesti e parole, che in sé hanno il potere di rendere presente, visibile e attuale la realtà menzionata. Ciò avviene durante una solenne celebrazione rituale. J. von Allmen afferma che l'anāmnesis è un richiamo rituale di un evento del passato per ridargli il suo potere originale e collocare coloro che fanno l'anämnesis nell'evento che viene ricordato nella celebrazione?

Il "memoriale" liturgico si orienta nel suo operare contemporaneamente su Dio e sull'uomo. È un'attività cultuale in cui l'Israele, ricordando le grandi opere realizzate da Dio per il suo popolo, pone queste opere davanti agli occhi di Dio, in modo che Egli si ricordi di ciò che ha fatto di persona per la salvezza di tutto il popolo. Dal fatto del ricordare da parte di Dio nasce una nuova realtà che diventa un aiuto efficace per l'uomo. Il ricordare di Dio equivale a un suo nuovo operare, sempre efficace e concreto.

${ }^{4}$ Cfr. J. Corbon, Pamięć [in:] Stownik Teologii Biblijnej, a cura di X. Léon Dufour, Poznań 1990, p. 640.

${ }_{5}^{5}$ Cfr. G. Ravasi, Deuteromonio [in]: Nuovo Dizionario di Teologia Biblica, a cura di P. Rossano, G. Ravasi, A. Girlanda, Cinisello Balsamo (Milano) 19945 , p. 394-396.

${ }^{6}$ Occorre notare che i termini greci usati nella versione dei LXX (Septuaginta) sono stati poi riportati nella terminologia novotestamentaria relativa al culto, specialmente all'Eucaristia. Cfr. D. Kwiatkowski, Teologiczno-liturgiczne znaczenie pojęć ,pamiętać” $i$,pamiątka” w Starym Testamencie, "Kaliskie Studia Teologiczne" 1 (2002), p. 128. [Occorre notare che i termini greci usati nella versione dei LXX (Septuaginta) sono stati poi riportati nella terminologia novotestamentaria relativa al culto, specialmente all'Eucaristia.

${ }^{7}$ Cfr. J. von Allmen, Saggio sulla cena del Signore, Roma 1968, p. 58. 
Questi due elementi che compongono il "memoriale" liturgico non sono separati l'uno dall'altro. In effetti, il ricordare Dio e ciò che Egli ha compiuto, significa mettersi alla Sua presenza oggi stesso "qui" e "ora". L'anāmnesis non è quindi solo un ricordo di un evento del passato, ma un evento reale che si rende presente. Nell'anāmnesis liturgica, l'evento salvifico trascende i confini del passato e diventa il presente 8 .

Il Libro del Deuteronomio, trattando del fatto di ricordare, non fa riferimento alla memoria oggettiva, ma alla memoria soggettiva, la quale attualizza ciò che ricorda in ogni concreta celebrazione cultuale. Usando i segni visibili, rende presente la realtà che era accaduta in passato. Non si tratta solo della salvezza data agli antenati che furono condotti fuori dalla schiavitù egizia e condotti attraverso il Mar Rosso, ma si tratta di qualcosa di più, qualcosa che è attuale al presente. Dio salva oggi, proprio in questo momento in cui viene ricordato un evento del passato: "Il Signore, nostro Dio, ha stabilito con noi un'alleanza sull'Oreb. Il Signore non ha stabilito quest'alleanza con i nostri padri, ma con noi che siamo qui oggi tutti vivi." (Dt 5,2-3). Questa frase è l'introduzione al kerygma del Libro del Deuteronomio (cfr. 4,1; 6,1). Sta avvenendo l'attualizzazione liturgica dell'alleanza. Pertanto l'alleanza non è un patto che appartiene solo al passato, ma una realtà che sta continuando, è presente e viva ${ }^{9}$.

Al cuore della "teologia del ricordo" del Deuteronomio c'è la seguente affermazione: "Ti ricorderai che sei stato schiavo nella terra d'Egitto e che il Signore, tuo Dio, ti ha riscattato; perciò, io ti dò oggi questo comando" $(15,15)$. Il ogetto focale della memoria di un Israelita è la storia della salvezza, che Dio stesso crea sin dall'inizio dell'esistenza di questa nazione. Nella memoria dell'Israele deve restare anche l'esperienza della schiavitù d'Egitto, cioè le pesanti persecuzioni e le sofferenze vissute in Egitto. Solo nel contesto delle sofferenze sperimentate si può apprezzare ciò che Dio ha compiuto conducendo fuori dalla terra della schiavitù ${ }^{10}$.

Dio non solo ha liberato il popolo dalla schiavitù d'Egitto, ma anche si è preso cura di lui e l'ha guidato attraverso il deserto verso la terra promessa. L'esodo dalla schiavitù è legato alle grandi opere che Dio ha fatto per salvare il suo popolo. E il popolo eletto non può dimenticarle, perché proprio è nel ricordarle che trova il potere di iniziare una nuova vita (Dt 4,9; 5,$15 ; 7,18 ; 8,2.18 ; 24,18.20 .22 ; 32,7)$.

${ }^{8}$ Cfr. J. Jeremias, Le parole dell'ultima cena, Brescia 1973, p. 309.

${ }^{9}$ Cfr. G. Ravasi, Deuteromonio, p. 395.

${ }^{10}$ Cfr. G. von Rad, Teologia Starego Testamentu, trad. B. Widła, Warszawa 1984, p. 182-186. Il Libro del Deuteronomio fa riferimento soprattutto alla Pasqua ebrea. Tuttavia le osservazioni in essa comprese, relative alla celebrazione della Pasqua come memoriale, si riferiscono a tutte le feste liturgiche dell'Israele. Cfr. ibid., p. 444-445. Il Libro del Deuteronomio fa riferimento soprattutto alla Pasqua ebrea. Tuttavia le osservazioni in essa comprese, relative alla celebrazione della Pasqua come memoriale, si riferiscono a tutte le feste liturgiche dell'Israele. Cfr. ibid. 
Comunque, per avere un quadro completo e per approfondire la fede, l'israelita dovrebbe anche ricordare le colpe e i peccati commessi contro il suo Dio (Dt 9,7).

Il mistero del rendere presente - attuallizzare (anämnesis) troviamo nell'ebraico rito pasquale. Nella pasquale Haggadah al Pesach, l'israelita esprime, tra l'atro, queste parole: "In tutte le generazioni, ognuno dovrebbe vedere in se stesso colui che è uscito dall'Egitto. Come è detto: In quel giorno tu spiegherai a tuo figlio: «È a causa di quanto ha fatto il Signore per me, quando sono uscito dall'Egitto» (Es 13,8)". Poiché non solo i nostri padri furono redenti dal Santo, sia benedetto, ma anche noi - insieme a loro. Come fu detto: "Ci fece uscire di là per condurci nella terra che aveva giurato ai nostri padri di darci" (Dt 6,23) ${ }^{11}$. Invece nel Talmud babilonese leggiamo:

Ecco, perché ci fu comandato di rendere grazie, proclamare, lodare, benedire, onorare, glorificare ed adorare Colui che ha compiuto questo miracolo per i nostri padri e per tutti noi che siamo stati condotti dalla schiavitù alla libertà, dalla sofferenza alla gioia, dal lutto alla festa, dall'oscurità alla sua piena luce, dalla schiavitù alla libertà $[\ldots]$ Alleluia $^{12}$.

L'unicità del culto israelitico non sta nei sacrifici, o nella celebrazione di varie festività, ma nel contenuto anamnetico, incluso nei sacrifici e in tutte le feste. Il culto è legato inscindibilmente con il ricordo degli eventi passati. Tutto ciò che costituisce il culto, quindi parole, gesti, rappresentazioni drammatiche, tutto ciò evoca le grandi opere compiute da Dio nel passato. Gli israeliti celebrano i fatti storici concreti dietro ai quali ci sta Jahvè. In effetti, Dio non si accontentò di stringere l'alleanza con un gruppo di persone che vivevano in un momento specifico. L'alleanza è una realtà che va fuori tempo e che abbraccia tutte le persone di ogni epoca. Per questo il popolo eletto celebra feste regolarmente ricordando gli eventi del passato. Le celebrazioni e i ricordi rendono questi eventi attuali e presenti. Le esortazioni profetiche e deuteronomiche sono accompagnate dalle esortazioni ancora più importanti, costituite dalle festività del calendario ebraico, che celebrano e attualizzano le azioni di Dio e, soprattutto, l'alleanza ${ }^{13}$.

Celebrando il ricordo degli eventi salvifici, non solo Israele, ma soprattutto Dio stesso si ricorda del suo popolo e, ricordando, rende la salvezza una realtà presente e attuale. Ogni celebrazione cultuale è un grande incontro del

${ }^{11}$ Hagada na Pesach [in]: Jak modla się Żydzi. Antologia modlitw, studio di M. Toma, (trad. Autore), Warszawa 2000, p. 160. In questa preghiera più volte viene accentuato il "qui" e 1' "ora" dell'evento pasquale. Durante la celebrazione si rendono presenti grandi opere, operate da Dio a favore d'Israele, durante la sua uscita dall'Egitto e il cammino vereso la Terra promessa — vedi ibid., p. 162-163, 165.

${ }_{12}$ Vedi S. de Diètrich, Boży plan zbawienia, (trad. Autore), Warszawa 1967, p. 45.

${ }^{13}$ Cfr. X. Leon Dufour, Condividere il Pane Eucaristico, Leumann-Torino 1983, p. 108. 
Dio dell'alleanza, il Dio che vive, opera ed è presente "qui e ora" con il popolo che partecipa al culto in una concreta celebrazione ${ }^{14}$. Il culto israelitico evoca le grandi opere di Dio, realizzate nel passato, rivela la loro potenza nel presente e nutre la speranza per un futuro e finale intervento salvifico di Dio. In questo senso, il culto è soprattutto un luogo di rivelazione, perché in esso si celebra, attualizza e realizza l'incontro di Dio con l'uomo. È anche un luogo di tradizione, perché attraverso il culto, la comunità tramanda di generazione in generazione ciò che Dio ha operato e continua a operare per tutto il popolo.

Riassumendo, occorrre far notare che il ricordare da parte di Dio è sempre efficace, fruttuoso e molto concreto nelle sue manifestazioni esteriori. Le opere che nascono dalla memoria di Dio scaturiscono dalla sua provvidenza e riguardano la storia della salvezza che è in un costante divenire. Va notato che qui non si trattata solo di un'operazione mentale, puramente speculativa. Dal fatto che Dio si ricordi dell'uomo deriva una situazione completamente nuova, sorge una nuova realtà: l'uomo che si trova nel bisogno, riceve un aiuto concreto. Il ricordare da parte di Dio è sempre un'azione attiva e creativa (cfr. Gn 9,15; Es 2,24-25; 6,5; Sal 105,8; 106,45; 111,5; Gdc 16,28; 2 Cr 6,42; 2 Re 20,3; Sal 74,2; 89,51; 103,14; 1 Mac 7,38; Ne 1,8; 5,19; 6,14; 13,14.22.29.32) $)^{15}$.

La Sacra Scrittura, e in particolare il Libro del Deuteronomio, tratta del passato del popolo eletto come di una realtà presente e attuale nella contemporaneità. Il verbo "ricordare" esprime qui la salvezza che sie rende presente nel culto. La rivelazione biblica presenta la storia come una storia d'amore, la storia dell'alleanza tra Dio e l'uomo. Il Dio dell'alleanza dona sempre all'uomo il suo infinito amore, e per questo l'uomo dovrebbe ricordarsi del suo Dio. Il ricordo delle grandi opere di Dio significava per l'uomo essere o non essere. Mentre Dio non si dimentica mai della sua alleanza, la memoria dell'uomo sembra molto debole e limitata. Nonostante le esortazioni del Libro del Deuteronomio: "Ma bada a te e guardati bene dal dimenticare le cose che i tuoi occhi hanno visto", l'uomo molto spesso si dimenticava del suo Dio (Dt 4,$9 ; 8,11 ; 9,7)^{16}$.

Per comprendere il "cuore" delle feste liturgiche ebraiche e cristiane, occorre guardarle e capirle come anamnesi, cioè il rendersi presente dell'azione salvifica di Dio nella storia. Il resto dell'articolo mostrerà in che modo i contenuti del Libro del Deuteronomio riguardanti la "memoria", intesa come il rendersi presente e l'attualizzazione, siano stati inclusi nell'attuale teologia dell'anno liturgico.

${ }^{14}$ Cfr. B. Neunheuser, Memoriale [in:] Nuovo Dizionario di Liturgia, a cura di D. Sartore, A.M. Triacca, Cinisello Balsamo (Milano) $1993^{5}$, p. 770-772.

${ }^{15}$ Cfr. ibid., p. 765-781.

${ }^{16}$ Cfr. J. Corbon, Pamięć..., p. 640. 


\section{L'anno liturgico come anamnesi degli eventi salvifici della vita di Cristo}

Le norme generali per l'ordinamento dell'anno liturgico e del calendario indicano al numero 1 il fondamento teologico dell'anno liturgico:

La santa Chiesa celebra, con sacro ricordo, in giorni determinati, nel corso dell'anno, l'opera di salvezza di Cristo. Ogni settimana, nel giorno a cui ha dato il nome di domenica, fa memoria della risurrezione del Signore, che ogni anno, insieme alla sua beata Passione, celebra a Pasqua, la più grande delle solennità ${ }^{17}$.

Dall'affermazione menzionata sopra risulta chiaro che al centro delle celebrazioni dell'anno liturgico si trova Gesù Cristo con il suo mistero pasquale. Inoltre, non si tratta solo di un ricordo storico degli eventi salvifici, ma del loro rendersi presenti, cioè l'anamnesi nel senso stretto della parola.

\subsection{Il mistero dell'anno liturgico - la persona e l'atto salvifico di Gesù Cristo}

La grandezza dell'anno liturgico consiste soprattutto nel suo stretto legame con il Misterium Christi, il quale collega con Dio e il suo piano salvifico realizzato pienamente in Cristo. Questo mistero reso attuale nella Chiesa, nella liturgia, costituisce il senso dell' anno liturgico, il quale si struttura secondo le tappe della missione di Gesù. Va sottolineato a questo punto che non si tratta qui di una semplice ricostruzione dei fatti ed eventi della vita di Gesù, ma soprattutto del riviverli sempre di nuovo e del vivere di essi ${ }^{18}$. L'anno liturgico è un'anamnesi nel senso pieno del termine. La liturgia celebra l'attualità del mistero. È il suo rendersi presente in diversi significati della parola: è una ripetizione di tipo rituale che ci pone di fronte a un evento, ma allo stesso tempo ci dona la forza del mistero celebrato. La liturgia ci pone nell'“oggi di Dio". Lo svelare del mistero di Cristo lungo tutto l'anno ha il carattere storico-salvifico ${ }^{19}$.

${ }_{17}$ Ogólne normy roku liturgicznego i kalendarza, nr 1 [in:] Mszał rzymski dla diecezji polskich, (trad. Autore), Poznań 1986, p. [66].

${ }_{18}$ Nella celebrazione dell'anno liturgico, sono importanti non tanto le date specifiche, quanto gli eventi che vengono celebrati. Per questo la Chiesa a volte, ad esempio, per motivi pastorali o liturgici, sposta le date di alcune festività, ad esempio il trasferimento della celebrazione della solennità dell'Ascensione alla 7a domenica di Pasqua, il trasferimento della solennità dell'Annunciazione del Signore, di S. Giuseppe, o della Beata Vergine Maria Regina di Polonia, se queste coincidono con le domeniche di Quaresima o le domeniche di Pasqua.

${ }^{19}$ Cfr. A. Bergamini, L'anno liturgico. Cristo festa della Chiesa. Storia, celebrazione, teologia, spiritualità, pastorale, Cinisello Balsamo (Milano) 2002, p. 40-47. 
Il Mistero di Cristo viene celebrato anche nei giorni festivi e nelle memorie di Maria, degli angeli e dei santi. Questo mistero diventa realtà sempre nella sua totalità. Tuttavia, a causa della limitata capacità di conoscere e di comprendere dell'uomo, questo mistero è stato disteso lungo tutto l'anno e viene presentato in vari aspetti. Attraverso la celebrazione dell'Eucaristia si realizza tutto il mistero di Cristo. Mentre le specifiche festività sottolineano la dimensione scelta di questo mistero ${ }^{20}$.

In questo contesto va sottolineato che l'anno liturgico ha non solo un significato pedagogico, ma è soprattutto la presenza, la presenza di Cristo e del suo mistero di salvezza dilatato nel tempo. Ne scrisse Papa Pio XII nell'enciclica Mediator Dei (1947): L'anno liturgico "non è una fredda e inerte rappresentazione di fatti che appartengono al passato, o una semplice e nuda rievocazione di realtà d'altri tempi. Esso è, piuttosto, Cristo stesso, che vive sempre nella sua Chiesa [...] [nei] misteri che sono perennemente presenti ed operanti" ${ }^{\prime 21}$.

\subsection{L'attualizzazione del mistero di "oggi” e "ora"}

Il liturgico "oggi" (lat. hodie) e "ora" (lat. nunc) non sono semplici datazioni, ma significano un mistero che si realizza, si rende presente qui e ora. Nel presente liturgico è inscritta una presenza che trascende il tempo. Abbiamo a che fare con il rendersi presente e attuale, che viene donato da Dio in modo disinteressato. Nella costituzione sulla liturgia Sacrosanctum Concilium leggiamo: "Ricordando in tal modo i misteri della redenzione, essa [la Chiesa] apre ai fedeli le ricchezze delle azioni salvifiche e dei meriti del suo Signore, le rende come presenti a tutti i tempi e permette ai fedeli di venirne a contatto e di essere ripieni della grazia della salvezza" ${ }^{22}$. La costituzione liturgica usa in questa affermazione il concetto caseliano di "mistero". La comprensione dell'anno liturgico come il rendersi presente dell'intero evento della salvezza è diventata possibile grazie al rinnovamento liturgico preconciliare, chiamato "Movimento liturgico"23. I Padri

${ }^{20}$ Cfr. B. Nadolski, Liturgika, t. 2: Liturgia i czas, Poznań 1991, p. 27-28.

${ }^{21}$ Pius XII, Encyklika o liturgii Mediator Dei, tłum. J. Wierusz-Kowalski, Kielce 1948, p. 90.

${ }^{22}$ CL 102.

${ }^{23}$ In particolare Odo Casel, presentando la liturgia come mistero, cioè come il rituale rendere presente l'opera salvifica di Cristo, in simile modo percepiva l'anno liturgico. Nella sua opera più importante, che ha posto le basi per la teologia liturgica conciliare e postconciliare, leggiamo: "La Chiesa vive nella fede e nei misteri del culto di Cristo. Questi misteri del culto sono il risultato e l'applicazione del mistero di Cristo. Dio, che si è rivelato per mezzo dell'Uomo-Cristo, opera anche dopo l'esaltazione di Cristo e proprio solo ora, per mezzo del Cristo - Sommo Sacerdote, continua a operare sulla terra, cioè secondo l'ordinaria economia della mediazione ecclesiale della grazia, attraverso il mistero del culto, il quale non è niente altro che il Dio-Uomo che continua ad operare sulla terra. Per questo esso porta anche, come pure il Dio-Uomo, il doppio carattere della Divina Maestà che è operante e del nascondimento sotto i simboli materiali, presi da questa terra, 
di questo Movimento vedevano ogni festa cristiana come un'attualizzazione dell'atto salvifico di Cristo. Partecipando alla festa, il credente entra effettivamente nella realtà della salvezza, cioè nell'incontro personale con Cristo ${ }^{24}$.

Il rendersi presenti dei misteri salvifici di Cristo avviene grazie alla presenza e l'azione dello Spirito Santo. Egli ravviva tutte le celebrazioni liturgiche, le ravviva e fa si che il mistero salvifico di Cristo si rende presente "oggi", "qui" e "ora" per "noi". La liturgia non mostra l'interesse solamente per il passato. Proprio al contrario, proclama che Dio opera le cose straordinarie nei nostri tempi. Celebriamo le feste liturgiche per vivere il nostro presente con Cristo ${ }^{25}$.

Il ruolo essenziale dell'anno liturgico consiste nell'aprire il tempo dell'uomo al tempo di Dio. Le feste liturgiche servono per collegare la nostra esistenza alla Sua esistenza, per assicurarci la nostra partecipazione agli eventi più importanti della vita di Gesù, affinché possiamo unirci ai suoi misteri. Essi aggiungono alla nostra vita la dimensione eterna. La loro celebrazione ci permette di respirare un'aura diversa e ci riempie della presenza di Dio.

\subsection{Il Cristo - "la pienezza del tempo" resa presente nella liturgia della Chiesa}

Con Gesù Cristo è giunta la pienezza del tempo, il tempo si è compiuto (cfr. Mc $1,15)$. In questo contesto, si può dire che il tempo terreno è stato colmato con il "tempo" di Dio, cioè con l'eternità. Partecipando al mistero di Cristo, celebrato nella liturgia durante tutto l'anno, l'uomo viene inserito in questa pienezza del tempo. L'uomo partecipa a questa pienezza del tempo in modo reale. È proprio grazie ai sacramenti l'uomo entra in contatto reale e attuale con il mistero di Cristo. Partecipando alle celebrazioni dell'anno liturgico, il credente riceve e sviluppa costantemente il dono di una vita nuova, che lo avvicina al compimento finale del tempo nella dimensione individuale ${ }^{26}$.

i quali allo stesso tempo nascondono e mostrano. Pertanto diventa possibile che il Signore, sebbene glorificato e manifesto per sempre in cielo, resti ancora nascosto sulla terra e ciò nonostante stia già sviluppando tutta la potenza della sua esaltazione. Per questo la presenza del Signore nei misteri occupa il posto di mezzo, tra la vita terrena di Cristo e il suo regno glorificato in cielo: la potenza di Dio è pienamente sviluppata, ma richiede ancora fede, non concede ancora una dimensione manifesta". O. Casel, Chrześcijańskie misterium kultu, tłum. M. Wolicki, Kraków 2000, p. 101.

${ }^{24}$ Cfr. M. Worbs, Chrystocentryzm roku liturgicznego wedtug Romana Guardiniego [in:] Misterium Chrystusa w roku liturgicznym. Księga pamiątkowa dedykowana Księdzu Profesorowi Bogustawowi Nadolskiemu TChr z okazji 55-lecia Święceń Kapłańskich i 50-lecia pracy naukowej, red. J. Nowak, Poznań 2012, p. 102-113.

${ }^{25}$ Cfr. B. Nadolski, Liturgika ..., p. 28-29.

${ }^{26}$ Cfr. D. Brzeziński, Chrystus wczoraj i dziś, i na wieki. Anamnetyczny wymiar roku liturgicznego, Torun 2010, p. 361. 
La continuazione della "pienezza del tempo" è il cosiddetto "tempo della Chiesa" in cui Cristo continua la sua missione salvifica ${ }^{27}$. In questo tempo succede il cosidetto fenomeno della ciclicità oppure la ripetizione dell'anno liturgico. Non si deve confondere la ripetizione delle feste liturgiche con il "ritorno" ciclico dei tempi di cui parlano altre religioni. Il tempo liturgico si ripete come una spirale che si srotola che tende alla parusia. L'anno liturgico riflette la vita di Dio e significa non solo la salvezza, ma rinnova e rende presente costantemente la vita di Dio. Il ritorno di alcuni elementi nella liturgia scaturisce dalla pienezza della vita e dell'amore divino. È ricevere la grazia che è incommensurabile e inesauribile. Anche se la grazia e l'amore si ripetono ciclicamente, tuttavia sono sempre diversi. Fa notare queste realtà Odo Casel, scrivendo: "Come la serpentina corre attorno circondando il monte, per raggiungere nel lento rialzarsi un'ardua vetta, così dovremmo ancora una volta ripercorrere il cammino a livello più alto, finché non ragginge il destino finale, Cristo stesso, il nostro fine" 28.

Il decorso dell'anno liturgico ricorda effettivamente una spirale. Tuttavia va notato che il suo percorso non riconduce allo stesso punto da capo, ma si muove in avanti, come l'attraversamento di un pendio del monte per raggiungere la vetta. C'è davvero un movimento circolare nell'anno liturgico, ma esso conduce il cristiano ad un continuo sviluppo spirituale, di cui fine ultimo è l'incontro con il Signore risorto nell'eternità ${ }^{29}$.

\section{Conclusione}

Lo scopo dell'articolo era quello di mostrare la dimensione anamnetica dell'anno liturgico. È un tema importante e attuale, soprattutto oggi, quando si osserva il comune fenomeno di laicizzazione delle festività, le quali prima avevano il carattere strettamente religioso. È più visibile nella commercializzazione e secolarizzazione del Natale e della Pasqua, nella cessazione di celebrazione della domenica come il giorno del Signore. Nella molteplicità di elementi e gadget invitanti a celebrare queste festività, quasi del tutto è scomparso il contenuto religioso. Si è arrivati a celebrare il Natale senza Gesù; a celebrare la Pasqua, ma senza la morte e la risurrezione di Gesù. Mentre ogni festa cristiana rende presenti gli eventi salvifici della vita di Gesù. Celebrando e partecipando alla festa,

${ }^{27}$ Cfr. CL 5-7.

${ }^{28}$ O. Casel, Chrześcijańskie misterium kultu ..., p. 165.

${ }^{29} \mathrm{Ha}$ descritto la visione dell'anno liturgico secondo Odo Casel in modo molto ampio, come l'attualizzazione del mistero di Cristo, Don Waldemar Pałęcki dell'KUL (Università Cattolica di Lublino). W. Pałęcki, Rok liturgiczny Pascha Chrystusa. Misterium roku liturgicznego wedtug Odo Casela OSB (1886-1948), Sandomierz 2006. 
il cristiano si immerge nel mistero della salvezza. Attraverso la festa entra in un rapporto profondo con Cristo e rafforza la sua fede.

Per tornare ad una corretta comprensione e al vivere le festività cristiane, è necessaria oggi una formazione liturgica più approfondita, che presenta l'anno liturgico come anamnesi della persona e dell'azione salvifica di Gesù Cristo. Il migliore luogo per tale formazione è la liturgia, perché è un evento storico-salvifico. Ben preparata e opportunamente celebrata, può diventare un luogo di mistagogia che introduce nei misteri dell'anno liturgico e in tutte le feste che lo compongono.

\section{The Book of Deuteronomy as a Theological Source for the Concept of Memorial (anamnesis) in the Liturgical Year}

\section{Summary}

The article discusses the concept of memorial (anamnesis) in relation to the liturgical year and argues that within the Old Testament theology the Book of Deuteronomy constitutes the main theological source for the concept. The liturgical calendar is not a simple calendar in which the dates of various feasts of the Church are indicated. It is important to note that the liturgical calendar presents the saving mystery of Jesus Christ, but in a manner spread over time. In order to understand the anametic dimension of the liturgical year, it is necessary to indicate the meaning of the feast in the Holy Scriptures. Especially, on the basis of the Book of Deuteronomy, also known as the "Book of Remembrance", it is shown that each biblical feast is an anamnesis, that is, the presence of a specific event in the history of salvation. This understanding of the feast also exists in the liturgy of the Church. The importance of the liturgical year comes primarily from the close relationship with the mystery of Christ. Each feast makes the "here" and "now" of the saving act of Jesus Christ present. It happens thanks to the presence and action of the Holy Spirit in the liturgy of the Church.

\section{Keywords}

Jesus Christ, Church, liturgy, liturgical year, anamnesis, making present, reminder, mystery 


\section{Księga Powtórzonego Prawa jako teologiczne źródło anamnetycznego wymiaru roku liturgicznego}

\section{Streszczenie}

Artykuł przedstawia anamnetyczny wymiar roku liturgicznego oraz jego starotestamentalne źródło teologiczne, którym jest Księga Powtórzonego Prawa. Kalendarz liturgiczny nie jest terminarzem, w którym zaznaczone są daty różnych świąt kościelnych. Jest on rozłożonym w czasie uobecnieniem misterium zbawczego Jezusa Chrystusa. Chcąc zrozumieć anamnetyczny wymiar roku liturgicznego, trzeba ukazać, czym było święto religijnej tradycji Izraela. Zwłaszcza na podstawie Księgi Powtórzonego Prawa, nazywanej „Księgą pamięci”, wykazano, że każde biblijne święto jest anamnezą, czyli uobecnieniem konkretnego wydarzenia $\mathrm{z}$ historii zbawienia. Takie rozumienie święta jest także w liturgii Kościoła. Znaczenie roku liturgicznego polega przede wszystkim na ścisłym powiązaniu go z misterium Chrystusa. Każde święto jest uobecnieniem „tu” i „teraz” zbawczego dzieła Jezusa Chrystusa. Dokonuje się ono dzięki obecności i działaniu Ducha Świętego w liturgii Kościoła.

\section{Słowa kluczowe}

Jezus Chrystus, Kościół, liturgia, rok liturgiczny, anamneza, uobecnienie, pamiątka, misterium

\section{Parole chiave}

Gesú Cristo, Chiesa, liturgia, anno liturgico, anamnesis, attualizzazione (far presente), memoria, mistero

\section{Bibliografia}

Allmen von J., Saggio sulla cena del Signore, Roma 1968.

Bergamini A., L'anno liturgico. Cristo festa della Chiesa. Storia, celebrazione, teologia, spiritualità, pastorale, Cisinello Balsamo (Milano) 2002.

Brzeziński D., Chrystus wczoraj i dziś, i na wieki. Anamnetyczny wymiar roku liturgicznego, Toruń 2010.

Casel O., Chrześcijańskie misterium kultu, tłum. M. Wolicki, Kraków 2000.

Concilio Vaticano II, La Costituzione sulla Sacra Liturgia Sacrosanctum Concilium, Città del Vaticano 2002, p. 48-78.

Corbon J., Pamięć [in:] Słownik Teologii Biblijnej, red. X. Léon Dufour, Poznań 1990, p. 639-642.

Diètrich de S., Boży plan zbawienia, Warszawa 1967.

Dufour Leon X., Condividere il Pane Eucaristico, Leumann-Torino 1983.

Jak modla się Żydzi. Antologia modlitw, studio di M. Tomal, Warszawa 2000.

Jeremias J., Le parole dell'ultima cena, Brescia 1973. 
Kwiatkowski D., Teologiczno-liturgiczne znaczenie pojęć „pamiętać” $i$ „pamiątka” w Starym Testamencie, „Kaliskie Studia Teologiczne” 1 (2002), p. 117-128.

Nadolski B., Liturgika, t. 2: Liturgia i czas, t. 2, Poznań 1991.

Neunheuser B., Memoriale [in:] Nuovo Dizionario di Liturgia, red. D. Sartore, A.M. Triacca, Cinisello Balsamo (Milano) $1993^{5}$, p. 765-781.

Ogólne normy roku liturgicznego i kalendarza [in:] Mszat rzymski dla diecezji polskich, Poznań 1986, p. [66-88].

Pałęcki W., Rok liturgiczny Paschą Chrystusa. Misterium roku liturgicznego wedtug Odo Casela (1886-1948), Sandomierz 2006.

Pius XII, Encyklika o liturgii Mediator Dei, tłum. J. Wierusz-Kowalski, Kielce 1948.

Rad von G., Teologia Starego Testamentu, tłum. B. Widła, Warszawa 1984.

Ravasi G., Deuteromonio [in:] Nuovo Dizionario di Teologia Biblica, a cura di P. Rossano, G. Ravasi, A. Girlanda, Cinisello Balsamo (Milano) 19945 , p. 391-397.

Tomal M. (studio), Come pregano gli Ebrei. Antologia delle preghiere, Warszawa 2000.

Worbs M., Chrystocentryzm roku liturgicznego wedtug Romana Guardiniego [in:] Misterium Chrystusa w roku liturgicznym. Księga pamiątkowa dedykowana Księdzu Profesorowi Bogusławowi Nadolskiemu TChr z okazji 55-lecia Święceń Kaptańskich i 50-lecia pracy naukowej, red. J. Nowak, Poznań 2012, p. 102-114. 